\title{
Working Memory Centered Interpreting Information Processing Study
}

\author{
Ke Yang, Jiang Xia, Liangsheng Wang \\ Southwest University for Nationalities, Chengdu, Sichuan 610041, China
}

\begin{abstract}
There are various factors affecting interpreting information transformation process and each factor restricts with each other. Working memory occupies an important memory in interpreting information process which along with complex emotion and anxiety is the most common one. Based on the comprehensive skills operation mechanism with the working memory as the core in interpreting cognitive development process, how working memory triggers anxiety emotional change should be studied in depth, how to control anxiety and keep high efficiency of interpreting information process with the working memory as the core during teaching and practicing should be noticed and then interpreting quality will be improved.
\end{abstract}

Keywords: working memory; interpreting competence; anxiety; psychological linguistics.

\section{Overview of Interpreting Information Processing}

Interpreting is a kind of information processing transformation process including language information, context information, cultural information, psychological information, emotional communication, aesthetic taste, customer recognition and other integrated communicative activities and the process interpreter understands information and interacts with environment. Interpretation has a higher demand of working memory, of which work memory capacity and working memory coordination mechanism is very important, directly restricting the interpreting information processing's speed and quality of each link. At the same time, changes in emotional experience largely affect the interpretation skills. From the perspective of psycholinguistic theory, it is necessary to analyze the three stages of input, intermediate processing and output of language information processing. In the field of language cognitive, according to the order of information processing research different dimensions are divided: regional accent English listening, schema and cross-cultural communication, working memory and professional language expression, which achieves stereoscopic perspective interpretation ability from the views of cognitive development and emotion.

In interpreting information processing, working memory plays an important role. Baddeley and Hitch put forward the concept of working memory in 1974 to illustrate its short-term storage and process. Working memory is described as human cognitive central and is one of the most active research field of cognitive psychology and cognitive neuroscience. People first mobilize the short-term memory when accepting outside information. Short-term memory capacity is very limited and its memory span is $7 \pm 2$ chunks. Working memory is different with short-term memory which simply responsible for information storage, because it bears the information storage and processing at the same time. It is also related to the complex cognitive skills of mankind, especially with the formation and improvement of skills such as language comprehension, vocabulary learning, reading comprehension, writing, logical reasoning and complex learning. The characteristics of working memory are closely related to the resolution of primitive information and the activities of translation output in the process of interpreting cognition. Therefore, the relationship between working memory and interpreting has become a concern in interpreting research. Zhang Wei (2007) The relationship between simultaneous interpretation and working memory is discussed roundly by using the method of comprehensive empirical method. Interpreting activities to promote the development of memory capacity to a certain extend. Simultaneous transmission process will interfere information which will impact memory effects. No significant relationships were observed between memory capacity and interpretation effect. The pressure of English to Chinese simultaneous memory is higher than that of Chinese to English simultaneous memory. In addition, the professional translator agreed that the language knowledge and interpretation skills are more important than memory capacity. At the same time, the working memory capacity of professional interpreters is significantly larger than that of other 
fields, and that of interpreters. Interpreting cognitive ability is not a static concept, but involves a series of conceptual subsystems and conceptual integration of subsystems' interaction and activation. Memory processing of interpreting is an important cognitive mechanism in the process of interpreting thinking, and there are certain different characteristics of alternate interpretation and simultaneous interpretation in memory processing. However, whether it is an alternate interpreter or simultaneous interpretation, its conceptuality nature of memory operation is the same. Working memory is an information transfer between memory containers, which belongs to the middle process of interpreting information processing, directly affecting the re-extraction of interpreting information and indirectly restricting the fluency of the whole process of language information processing.(Zhong Jie, 2010: 136-137) In 2011, an experiment summarized that working memory and interpretation skills would have a positive impact on simultaneous interpretation. The advantage is that it adds the content of interpretation, which reflects the important impact of working memory on simultaneous interpretation, but it is not mentioned about both working memory and interpreting skills. And Chen Liwen (2011) believed that the memory processing of interpretation is an important cognitive mechanism of the process of interpretation. Although it reflects distinguishing characteristics consecutive and simultaneous interpretation in the memory processing program, the conceptual nature of their memory operation is the same.

\section{Relationship Between Working Memory and Anxiety Dimension}

Because of the complexity of the complex information exchange work of interpretation, more and more theoretical researchers began to attach importance to the anxiety of interpretation skills. Anxiety is one of the emotional states, and it is a psychological state that many interpreters have shown before the spot. Interpreting anxiety is more prevalent among student translators and professional translators. Excessive tension will interfere with human perception, memory, thinking and other awareness activities. But moderate tensions help the translator be focus and stimulate the translator to mobilize the body resources to do the interpreting work. Therefore, maintaining moderate tensions is one of the essential conditions for the completion of interpreting tasks. And most of the anxiety is also related to human personality, so we cannot force them to change. Therefore, translators can only use their own personality to adjust anxiety, transforming it into power and concentration. Anxiety is a typical factor in the impact of affective factors on the quality of interpretation, then what is the proportion of each stage of input, intermediate processing, and output information processing? Kang Zhifeng proposed the level of interpretation anxiety. The study found that the relationship of interpreting professionals' anxiety (stress) control, short-term memory and rapid response and the performance of interpretation that only rapid response significantly affects the interpretation of performance. Through multiple comparisons, it was found that the interpreting with lower three levels of interpreting abilities was significantly worse than that of the general and the high level. At the same time, it will lost or be unable to convert information, resulting in information output failure or even being unable to speak out. Low anxiety and high anxiety will equally interfere with the brain's cognitive process, prolong the reaction time of the brain, and lead speaking with hesitation. The results of the multiple comparisons further show the differences in the interpretation performance of the students with different anxiety levels and the breadth of working memory. Students with high degree of anxiety are of low breadth of memory, and students with low degree of anxiety are of high breadth of memory. In order to gain a deeper understanding of the impact of anxiety on interpretation memory, the following studies were conducted. What dimensions does interpreting information processing have? At present, there are: bilingual proficiency, English accent of different areas, working memory, background knowledge, schema, cross-cultural communication, language expression skills norms, mastering of interpretation strategies, adaptable skills, interpretation experience, emotional emotions, etc. There is also a complex interaction between each dimension. In various dimensions, the research results based on interpretation work memory and the latest research achievements of foreign language research field have been comprehensively sorted out in this paper, and The empirical study of language proficiency (bilingual 
ability) and emotional dimension (interpretation anxiety) have been made based on the relevant theory, practical observation and the objective data. At the same time, combined with the working memory test method, interpreting anxiety psychological measurement methods and the mechanism research method of working memory on the interpretation anxiety, research programs were carried out and data results were analyzed to draw conclusions. The cognitive process of interpretation is a heavy load, and a high-skilled activity with high concentration, fast operation, information real-time processing. Therefore, a qualified translator should have some special psychological characteristics, if not meet the specific psychological requirements, interpretation anxiety followed by will affect normal interpretation work. The theoretical definition of working memory and interpretation anxiety, especially the latter, should describe its connotation clearly based on previous studies, being accurate and representative.

\section{Conclusion}

Interpreting research needs to be closely integrated with the specific professional operation, the analysis of actual data of professional interpretation should be taken full account in the academic point of view; the actual information processing links, emotional changes, testing certification links should be controlled more from the theoretical point of view into the interpretation. Interpreting ability Interdepartmental research and cross-interface interpretation capacity multi-dimensional decomposition has important application value. In the structure of interpretation competence which with the working memory as the core, the causality and interaction cognitive and emotion, is the working memory (on behalf of cognitive) triggering anxiety (on behalf of emotion), or anxious triggering the working memory. This paper deeply studied cognitive development and emotional change represented by anxiety, and further from the view of cognitive and emotional theory analyzed the mechanism of working memory to interpretation anxiety and then the interpretation quality. In short, the research of the role of working memory on interpretation anxiety and cognition to interpretation emotion is still relatively less. This is a new attempt. If the research of this area is broken through, whether for interpretation teaching or interpretation test, it will have a great reference value.

\section{Acknowledgements}

Fund Project: Supported by the Fundamental Research Funds for the Central Universities, Southwest University for Nationalities), Project Number: 2014SZYQN84, Project Name: The Mechanism Study of Working Memory to Interpretation Anxiety; And supported by research funds by Education, Science and Technology Department of State Ethnic Affairs Commission of the People's Republic of China, Project Number: 14XNZ006, Project Name: The Study of Dimensions in the Interpreting Information Processing Processes

\section{References}

[1] Zhang Wei, Wang Kefei (Beijing Foreign Studies University Beijing 100089) Research of interpretation and working memory [J]. Foreign Languages and Teaching, 2007, 1.

[2] Zhong Jie, Baddeley. Review of working memory model development [J]. Journal of Hubei Economy Academy. 2010, (24)4. .

[3] Zhan Liwen. Meaning negotiation model of interpretation thinking process [M]. China Social Sciences Publishing House, 2011, 12. 